\title{
GEOLOGICAL MAPPING OF PLUTO AND CHARON USING NEW HORIZONS DATA
}

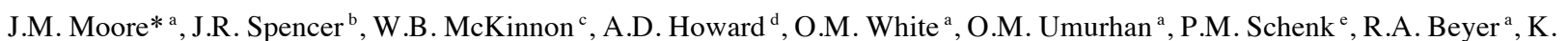 \\ Singer $^{\text {b }}$, S.A. Stern ${ }^{\text {b }}$, H.A. Weaver ${ }^{\mathrm{f}}$, L.A. Young ${ }^{\mathrm{b}}$, K. Ennico Smith ${ }^{\mathrm{a}}$, C. Olkin ${ }^{\mathrm{b}}$, and the New Horizons Geology and Geophysics \\ Imaging Teamz
}

\begin{abstract}
a NASA Ames Research Center, Moffett Field, CA, 94035, (jeff.moore@nasa.gov), ${ }^{\mathrm{b}}$ Southwest Research Inst., 1050 Walnut St.,

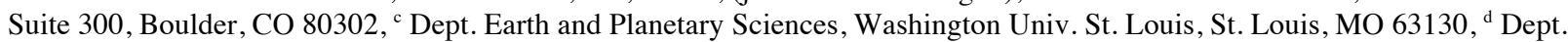
Environmental Sciences, University of Virginia, Charlottesville, VA 22904, e Lunar and Planetary Inst., 3600 Bay Area Blvd., Houston, TX 77058, ${ }^{\mathrm{f}}$ Johns Hopkins University Applied Physics Laboratory, Laurel, MD 20723
\end{abstract}

\section{Commission IV, WG IV/8}

KEY WORDS: Pluto, Charon, Geological Mapping, Spatial Databases

\begin{abstract}
:
Pluto and Charon exhibit strikingly different surface appearances, despite their similar densities and presumed bulk compositions. Systematic mapping has revealed that much of Pluto's surface can be attributed to surface-atmosphere interactions and the mobilization of volatile ices by insolation. Many mapped valley systems appear to be the consequence of glaciation involving nitrogen ice. Other geological activity requires or required internal heating. The convection and advection of volatile ices in Sputnik Planum can be powered by present-day radiogenic heat loss. On the other hand, the prominent mountains at the western margin of Sputnik Planum, and the strange, multi-km-high mound features to the south, probably composed of $\mathrm{H}_{2} \mathrm{O}$, are young geologically as inferred by light cratering and superposition relationships. Their origin, and what drove their formation so late in Solar System history, is under investigation. The dynamic remolding of landscapes by volatile transport seen on Pluto is not unambiguously evident in the mapping of Charon. Charon does, however, display a large resurfaced plain and globally engirdling extensional tectonic network attesting to its early endogenic vigor.
\end{abstract}

\subsection{Introduction}

The New Horizons spacecraft flew past the Pluto system on 14 July 2015, and provided our first view of the geology of Pluto and Charon by obtaining hundreds of images, including extensive stereo, with hemispheric coverage at best resolution $0.47 \mathrm{~km} / \mathrm{pixel}$ and regional coverage at resolutions down to 80 meters/pixel on Pluto. The best imaging covered the antiCharon hemisphere of Pluto and the Pluto-facing hemisphere of Charon, but global context was provided by approach imaging of all longitudes at resolutions of $20 \mathrm{~km} /$ pixel or better. Regions south of $38^{\circ} \mathrm{S}$ were in permanent darkness, though illumination by Pluto's haze extended useful imaging several $100 \mathrm{~km}$ into the dark regions on the encounter hemisphere. Almost all highresolution imaging has now been returned to Earth. Preliminary landform analysis results are published (Stern et al., 2015; Moore et al., 2016) and can be seen in the physiographic map of Figure 1.

\subsection{Pluto}

Pluto shows astonishing geological variety (Fig. 1). Much of its surface is ancient and relatively heavily cratered (Fig. 2), with craters up to $250 \mathrm{~km}$ in diameter and one possible $900 \mathrm{~km}$ impact basin (Singer et al., 2016). However most of the cratered terrains have been heavily modified. Mantles up to a few $\mathrm{km}$ thick partially cover much of the northern hemisphere, and are bounded by complex scarps. Craters and mantles are locally heavily eroded by processes that form fluted slopes and interconnected, sometimes dendritic, valleys. The nature of the mantling and erosion is difficult to decypher, particularly given Pluto's current low atmospheric pressure of $\sim 10 \mu$ bars which limits the effectiveness of aeolian erosion and sediment transport: we suspect glacial or sub-glacial processes are involved. The mantles are unlikely to be primarily composed of $\mathrm{N}_{2}$ condensed from the atmosphere, which could not support the observed topography (Brown and Kirk, 1994). Tectonism on the encounter hemisphere is limited to sparsely distributed extensional graben.

The encounter hemisphere is dominated by a $3 \mathrm{~km}$ deep basin of possible impact origin, containing an $\sim 900-\mathrm{km}$ diameter plain of smooth bright material, Sputnik Planum (all feature names here are informal). As the geological map of the surface of Sputnik Planum (Fig. 3) illustrates, there are no detectable impact craters and is thus extremely young, probably $<10 \mathrm{Ma}$ old (White et al., 2016; Moore et al., 2016; Greenstreet et al., 2015). The northern two-thirds of Sputnik Planum is dominated by a network of polygons resembling convection cells, and shows obvious flow features along its margins, while the southern part is punctuated by vast swarms of aligned km-scale pits. We interpret Sputnik Planum to be a deep, convecting, deposit of low-viscosity volatile ices, probably dominated by $\mathrm{N}_{2}$ (consistent with its surface composition, (Grundy et al., 2016)) that has accumulated within the basin.

On the western margin of Sputnik Planum is an intermittent chain of rugged mountains composed of jumbled blocks up to 5 $\mathrm{km}$ high (See map in Fig. 3) (White et al., 2016), which in many places resemble Europan chaos and appear to have formed by disruption and transport of pre-existing crust, perhaps due to undermining by volatile ices from Sputnik Planum. The height and steepness of the mountains, and their near infrared spectra (Grundy et al., 2016) imply a water ice composition. Elsewhere, Sputnik Planum is surrounded by even more baffling features. These include a $3-4 \mathrm{~km}$ high putative

* Corresponding author 
cryovolcano (Wright Mons, bottom of Fig. 3), elongated hills covered in aligned $300-\mathrm{m}$ high blade-like features (Tartarus Dorsa), and extensive pitted terrain cut by what appear to be active glaciers flowing into Sputnik Planum (White et al., 2016).

\subsection{Charon}

Charon is also geologically varied, though all terrains are heavily cratered and most appear to be roughly $4 \mathrm{Ga}$ old. The northern portion of the encounter hemisphere is extremely rugged (Fig. 4), with craters up to $240 \mathrm{~km}$ in diameter and a polygonal network of broad troughs up to $10 \mathrm{~km}$ deep. A series of parallel graben and tilted blocks separate the northern terrain from much smoother plains to the south. Convex marginal scarps, 1-2 km high, some of which form moats around isolated mountains, suggest that the material that surfaced the plains was a viscous fluid, perhaps an ammonia/water mixture. Many young craters show conspicuous light and dark rays, suggesting subsurface inhomogeneities.

\subsection{Implications}

Despite extensive recent activity around Sputnik Planum, much of the Pluto's surface is ancient, having undergone only surficial modification over the past $4 \mathrm{Ga}$. This is in striking contrast to Triton where ongoing activity appears to have erased large craters globally (Schenk and Zahnle, 2007). Triton's much younger mean surface age suggests that its activity is powered by additional heat sources not available to Pluto, probably heating by obliquity tides (Nimmo and Spencer, 2015). Pluto's radiogenic and residual primordial heat is nevertheless able to maintain recent and ongoing activity in Sputnik Planum and its environs, perhaps due to the lower heat flow necessary to mobilize $\mathrm{N}_{2}$ and other volatile ices compared to water ice. The age and nature of Charon's activity is comparable to that of similar-sized giant planet moons such as Ariel (Jankowski and Squyres, 1988) and likely postdates any likely tidal heating from post giant-impact orbital evolution (Barr and Collins, 2015). This suggests that tidal heating may not be necessary to produce similar ancient activity seen on many icy moons.

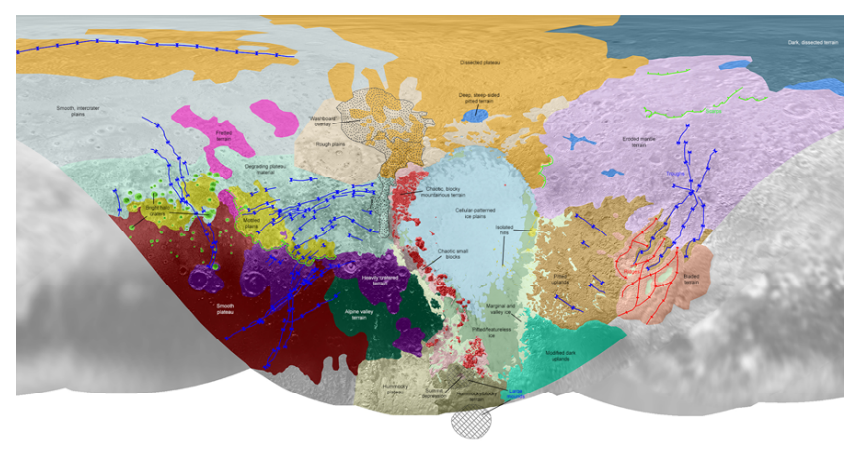

Figure 1. Simple cylindrical global projection of a physiographic province map for Pluto. $180^{\circ}$ longitude is located at the center of the figure. This figure should NOT be interpreted as a geological map, rather it provides a general indication of where terrains have been recognized on Pluto. Terrains within low-resolution zones are not mapped.

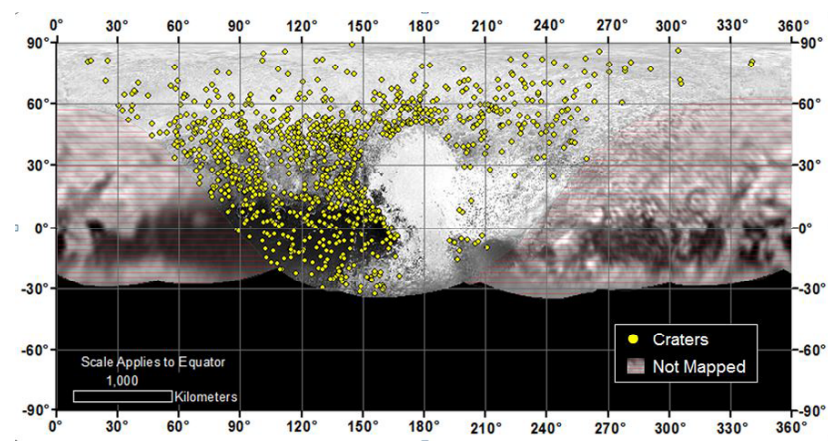

Figure 2. Crater Distribution Map of Pluto. Locations of more than 1,000 craters mapped on Pluto by NASA's New Horizons mission indicate a wide range of surface ages, which likely means that Pluto has been geologically active throughout its history (Singer et al., 2016).

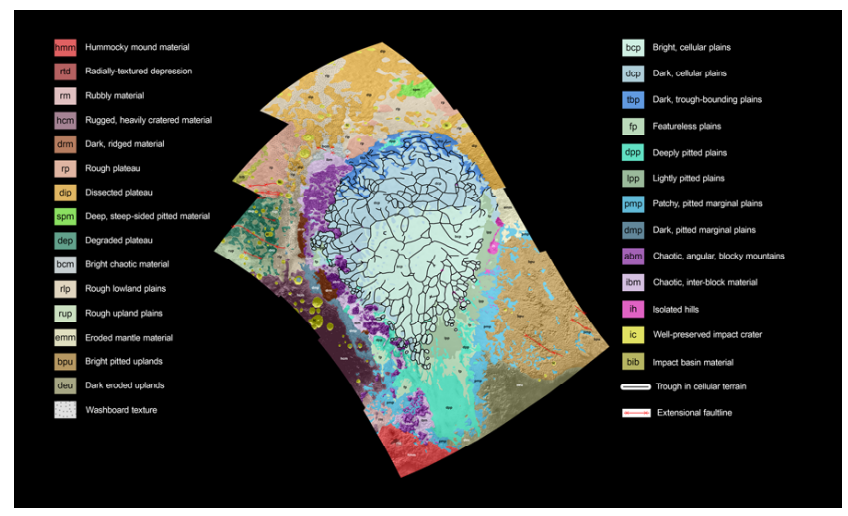

Figure 3. This geological map covers a portion of Pluto's surface that measures 2,070 kilometers from top to bottom, and includes the vast nitrogen-ice plain informally named Sputnik Planum and surrounding terrain. The key explains the colors that represent different geological terrains. Each terrain, or unit, is defined by its texture and morphology - smooth, pitted, craggy, hummocky or ridged, for example. How well a unit can be defined depends on the resolution of the images that cover it. All of the terrain in this map has been imaged at a resolution of approximately 320 meters per pixel or better, meaning scientists can map units with relative confidence. The various blue and greenish units that fill the center of the map represent different textures seen across Sputnik Planum, from the cellular terrain in the center and north, to the smooth and pitted plains in the south. The black lines represent the troughs that mark the boundaries of cellular regions in the nitrogen ice. The purple unit represents the chaotic, blocky mountain ranges that line Sputnik's western border, and the pink unit represents the scattered, floating hills at its eastern edge. The possible cryovolcanic feature informally named Wright Mons is mapped in red in the southern corner of the map. The rugged highlands of the informally named Cthulhu Regio is mapped in dark brown along the western edge, and is pockmarked by many large impact craters, mapped in yellow. The base map for this geologic map is a mosaic of 12 images obtained by the Long Range Reconnaissance Imager (LORRI) at a resolution of 390 meters per pixel (White et al., 2016). 


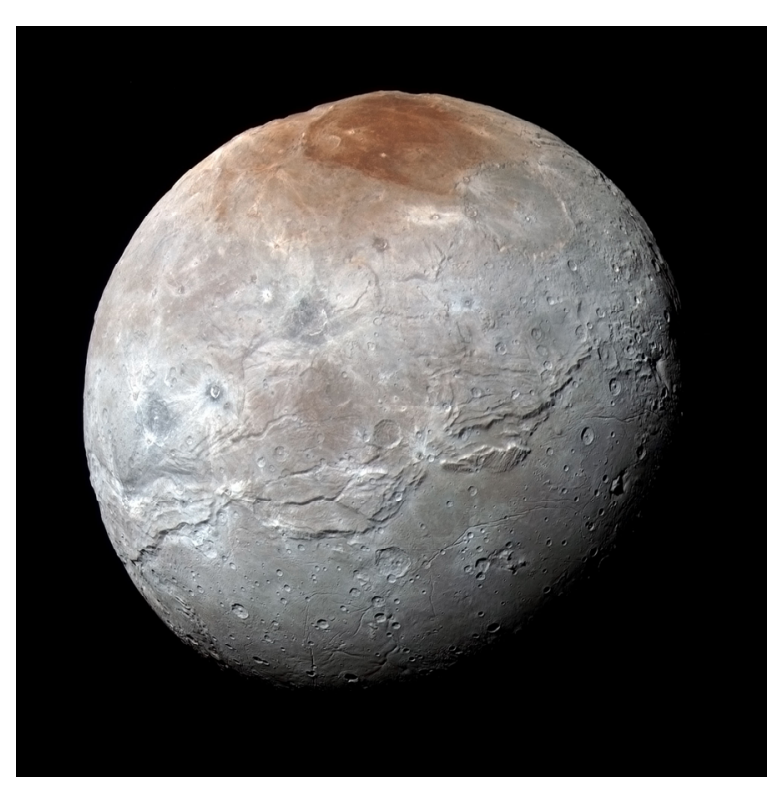

Figure 4. Encounter Hemisphere of Charon. This is a highresolution enhanced color view of Charon taken near closest approach. The image combines blue, red and infrared images taken by the spacecraft's Ralph/Multispectral Visual Imaging Camera (MVIC); the colors are processed to best highlight the variation of surface properties across Charon. Charon's color palette is not as diverse as Pluto's; most striking is the reddish north (top) polar region, informally named Mordor Macula. Charon is 1,214 kilometers in diameter; image resolution is 1.4 $\mathrm{km}$ per pixel.

\section{ACKNOWLEDGEMENTS}

This work was supported by NASA's New Horizons project.

\section{REFERENCES}

Barr, A.C. and Collins, G.C., 2015. Tectonic activity on Pluto after the Charon-forming impact. Icarus, 246, 146-155.

Brown, R.H. and Kirk, R.L., 1994. Coupling of volatile transport and internal heat flow on Triton. J. Geophys. Res., 99, 1965-1981.

Greenstreet, S., Gladman, B., McKinnon W.B., 2015. Impact and cratering rates onto Pluto. Icarus, 258, 267-288.

Grundy, W. M., et al., 2016. Surface compositions across Pluto and Charon, Science 351, aad9189. DOI: 10.1126/science.aad9189

Jankowski, D.G. and Squyres, S.W., 1988. Solid-state ice volcanism on the satellites of Uranus. Science, 241, 1322-1325.

Moore, J.M. et al., 2016. The geology of Pluto and Charon through the eyes of New Horizons. Science, 351, aad7055, 1284-1293. DOI: $10.1126 /$ science.aad7055

Nimmo F. and Spencer J.R., 2015. Powering Triton's recent geological activity by obliquity tides: Implications for Pluto geology. Icarus, 246, 2-10.
Schenk, P.M., Zahnle, K., 2007. On the negligible surface age of Triton. Icarus, 192, 135-149.

Singer, K.N., et al., 2016. Craters on Pluto and Charon Surface Ages and Impactor Populations. 47th Lunar and Planetary Science Conference, The Woodlands, Texas \#1903, p.2310.

Stern, S. A., et al., 2015. The Pluto system: Initial results from its exploration by New Horizons. Science, 350, aad1815. DOI: $10.1126 /$ science.aad 1815

White, O.L., et al., 2016. Geomorphological Mapping of the Encounter Hemisphere on Pluto. 47th Lunar and Planetary Science Conference, The Woodlands, Texas, \# 1903, p.2479. 